\title{
Ultrasensitive electrolyte-assisted temperature sensor
}

\author{
Mina Shiran Chaharsoughi ${ }^{1}$, Jesper Edberg $\mathbb{D}^{2}$, Peter Andersson Ersman $\mathbb{D}^{2}$, Xavier Crispin ${ }^{1}$, Dan Zhao $\mathbb{D}^{1 凶}$ and \\ Magnus P. Jonsson (iD ${ }^{1 凶}$
}

Heat sensors form an important class of devices that are used across multiple fields and sectors. For applications such as electronic skin and health monitoring, it is particularly advantageous if the output electronic signals are not only high, stable, and reproducible, but also self-generated to minimize power consumption. Here, we present an ultrasensitive heat sensing concept that fulfills these criteria while also being compatible with scalable low-cost manufacturing on flexible substrates. The concept resembles a traditional thermocouple, but with separated electrodes bridged by a gel-like electrolyte and with orders of magnitudes higher signals (around $11 \mathrm{mV} \mathrm{K}^{-1}$ ). The sensor pixels provide stable and reproducible signals upon heating, which, for example, could be used for heat mapping. Further modification to plasmonic nanohole metasurface electrodes made the sensors capable of also detecting light-induced heating. Finally, we present devices on flexible substrates and show that they can be used to detect human touch.

npj Flexible Electronics (2020)4:23; https://doi.org/10.1038/s41528-020-00086-5

\section{INTRODUCTION}

The ability to detect heat and temperature is essential in nature, and has aided animals, plants, and microorganisms to find their optimal conditions to grow and avoid damaging extremes ${ }^{1,2}$. For humans, our skin allows detection of local temperature changes induced both by contact with warm objects and via exposure to light radiation ${ }^{3,4}$. Heat sensing thereby forms an essential part of the skin's integrated network of sensors, which aid our interaction with the outside world by enabling detection of external stimuli ${ }^{4}$. Motivated by applications such as humanoid robotics, prosthetics, and wearable electronics, recent research has devoted significant efforts to develop electronic skin (e-skin) that can mimic the functionalities of human $\mathrm{skin}^{5-9}$. For such e-skin, augmentation with heat sensing is one of the critical requirements ${ }^{10-13}$. Previous research has explored different heat sensing principles for e-skin, including heat-induced resistance changes of conducting materi$\mathrm{als}^{14}$, variations in source-drain current of transistors ${ }^{15}$, and frequency-changes in piezoelectric devices ${ }^{16}$. Achieving high sensitivity for such sensors often involves microfabrication or patterning that are rather complicated and expensive in terms of large-scale production of low-cost sensors ${ }^{14}$. Furthermore, the above examples require an external electrical current to be applied through the devices in order to generate the sensor signal, which means consumption of energy ${ }^{14}$. Interestingly, human skin itself is a low-energy consuming sensory system ${ }^{8}$, which can inspire the design of self-powered sensors that generate output signals without external power ${ }^{17,18}$.

Pyroelectric systems are known to self-generate electrical signals during changes in temperature. A change in temperature affects the self-polarization over the pyroelectric material, leading to electrical currents to compensate for those changes ${ }^{19-21}$. However, pyroelectric signals are transient and quickly decay to zero after a change in temperature has taken place, which is not suitable for long-term temperature monitoring ${ }^{22-25}$. By contrast, recently developed ionic thermoelectric sensors ${ }^{26}$ can provide self-generated open-circuit voltages that are stable over time. A drawback is that ionic thermoelectric voltages do not correspond only to the temperature of a single target sensor spot, but they are induced by a temperature gradient between two different positions ${ }^{27}$. No signal is obtained upon uniform heating of the whole material or device. The requirement of a difference in temperature over the material poses challenges in terms of monitoring absolute temperatures of single spots, in particular for small dimensions and thicknesses where the whole material is heated. So far, the most commonly used self-powered temperature sensor is the thermocouple (TC), which provides thermovoltages based on the difference in Seebeck coefficients of two electronic conductors forming a junction ${ }^{28}$. However, the sensitivity of TCs is limited to tens of microvolts per kelvin due to low electronic Seebeck coefficients ${ }^{29}$. Sensors that provide higher signals for the same heat stimulus could not only improve resolution, but also open up for concepts that benefit from large absolute changes in voltage, such as heat-gated transistors ${ }^{30}$.

Here, we present a concept for self-powered heat sensing that resembles a traditional TC, but with orders of magnitude higher sensitivity. The so-called electrolyte-assisted temperature sensor (EATS) does not require any spatial temperature differences and can therefore be thin. EATS provides large and stable signals (not only transient signals) that are proportional to changes in temperature (from 14 to $55^{\circ} \mathrm{C}$ ). The large signals make EATS suitable for detection of small temperature changes $\left(0.1^{\circ} \mathrm{C}\right)$, with response better than commercial TCs. The sensor signals are reproducible and the devices are stable over long periods (at least 6 months). The concept has a simple structure composed of two different metals connected by a printable gel-like electrolyte, and is suitable to be applied on various rigid or flexible substrates by low-cost manufacturing methods. Furthermore, we present a modified type of EATS for detection of light-induced heating. For these modified devices, the metal electrodes are perforated with nanoholes, which make them interact strongly with light by conversion of incident light to plasmons (collective charge oscillations in the metal). A significant fraction of the plasmons decay via absorption and local heat generation ${ }^{31,32}$, which increases the temperature and induces signals in the sensor. The

${ }^{1}$ Laboratory of Organic Electronics, Department of Science and Technology, Linköping University, SE-60174 Norrköping, Sweden. ${ }^{2}$ RISE Research Institutes of Sweden, Bio- and

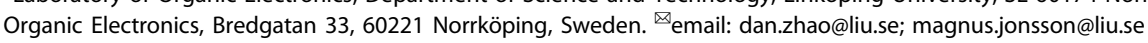




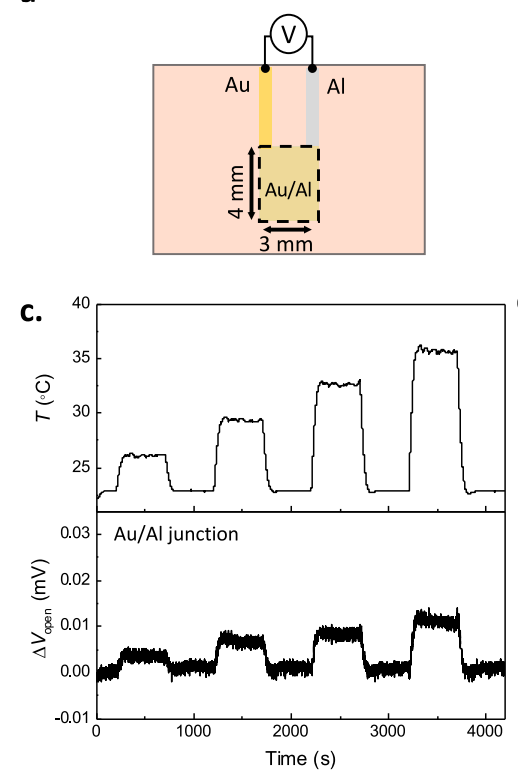

c.

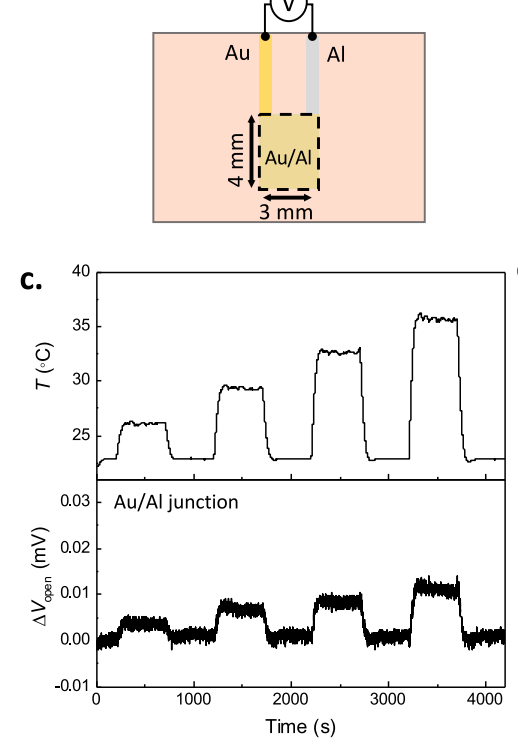

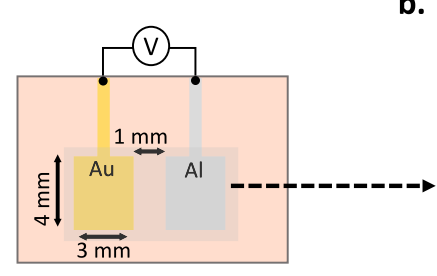

b.

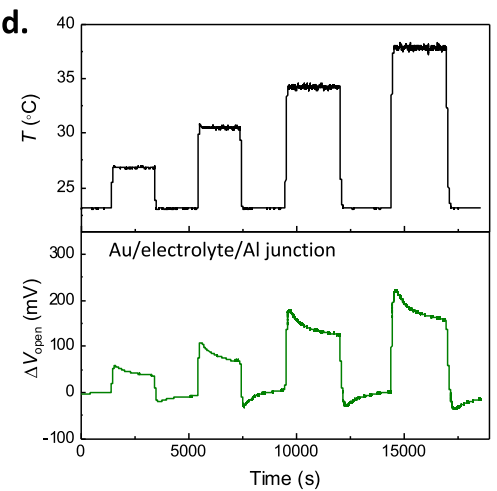

e.
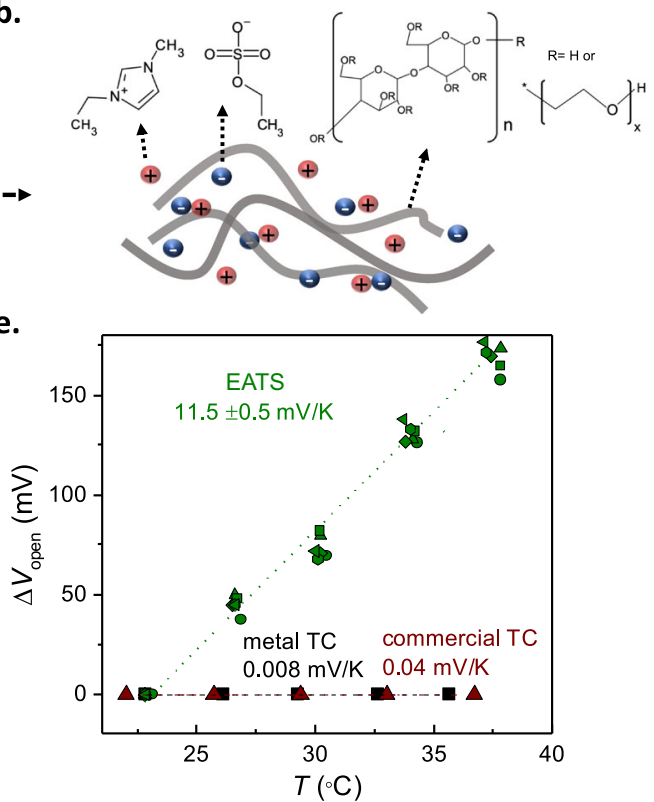

Fig. 1 Demonstration of the EATS and comparison with traditional TCs. a Schematic illustration of an Al/Au junction (left) and the EATS device (right). The Al/Au junction device consists of $20 \mathrm{~nm}$-thick aluminum and gold layers overlapping in a $12 \mathrm{~mm}^{2}$ area. For the EATS device, the $\mathrm{Au}$ and $\mathrm{Al}$ electrodes are separated and bridged by an electrolyte (shaded region, with areas overlapping with the electrodes of $12 \mathrm{~mm}^{2}$ ). b Schematic illustration of the gel-like electrolyte used in EATS a and the chemical structures of the compositions in the gel. c Variations in open-circuit voltage of an $\mathrm{Al} / \mathrm{Au}$ junction (bottom panel) upon controlled temperature changes (top panel). $\mathbf{d}$ Variation in open-circuit voltage of an EATS (bottom panel) upon controlled temperature changes (top panel). e Change in open-circuit voltage versus temperature for six EATS devices and comparison with the $\mathrm{Al} / \mathrm{Au}$ junction (black squares) and a commercial TC (red triangles). Different green symbol shapes indicate the results from different samples, and the error is the standard deviation from these six samples. The results were obtained in a cleanroom with relative humidity $(\mathrm{RH})$ of $30 \%$.

principle of using thermoplasmonic nanohole films to convert light to electrical signals follows our previous work on radiationinduced heat sensing ${ }^{33-35}$. Finally, we evaluate the EATS concept for heat mapping using sensor pixel arrays, and demonstrate its capability to detect human touch.

\section{RESULTS AND DISCUSSION}

Demonstration of the concept and comparison with traditional TCs

Figure 1 demonstrates the concept of the EATS, based on two separated gold $(\mathrm{Au})$ and aluminum $(\mathrm{Al})$ electrodes connected via an electrolyte (see the rightmost sketch in Fig. 1a). We chose a gel electrolyte based on an ionic liquid (1-ethyl-3-methylimidazolium ethylsulfate, [EMIM][ESO $\left.{ }_{4}\right]$ mixed with 2-hydroxyethyl cellulose (HEC, see Fig. 1b) as the gelling agent. This electrolyte is compatible with screen printing for roll-to-roll device manufacturing and also maintains constant concentration under long-term heating due to its low vapor pressure in the studied temperature range $^{36-38}$.

As shown in Fig. 1d, varying the temperature ( $T$, top panel) of the EATS led to large changes in the open-circuit potential between the two electrodes ( $\Delta V$, bottom panel). These results were reproducible between devices, as clear from Fig. 1e which presents $\Delta V$ as a function of $T$ for six different EATS devices. Linear fitting gives an average temperature sensitivity $(\mathrm{d} V / \mathrm{d} T)$ of $11.5 \mathrm{mV} \mathrm{K}^{-1}$ with standard deviation of $0.5 \mathrm{mV} \mathrm{K}^{-1}$. Likewise, the same EATS device responding to (24) multiple cycles of fixed temperature stimuli showed repeatability of the signals (Supplementary Fig. 1).

As comparison, we prepared simplified TC devices without electrolyte for which the Al and Au electrodes were connected (Al/ $\mathrm{Au}$ junction) (see the leftmost sketch in Fig. 1a). Such a device provided only small changes in open-circuit voltage upon temperature changes (Fig. 1c), with almost four orders of magnitude lower sensitivity (Fig. 1e). Compared to the commonly used commercial K-type TC (based on $\mathrm{NiCr}-\mathrm{Ni}$ ) with temperature sensitivity of $0.04 \mathrm{mV} \mathrm{K}^{-1}$ (Fig. 1e), the EATS has 275 times higher sensitivity. Monitoring the temperature at equilibrium (without temperature steps) shows that the proof-of-concept EATS sensor provides 4-5 times lower fluctuations than the optimized commercial TC (see Supplementary Fig. 2). The response time for the commercial TC (to reach stable values) varied between 90 and $150 \mathrm{~s}$ depending on the magnitude of the temperature change. For the EATS, the response time (to reach the peak value) was around 150-220 s, which we expect can be improved through further optimization of the concept.

Investigation of the heat sensing mechanism

In discussing the mechanism of the sensor concept, we note that the surface potential of metal electrodes can vary with temperature via variations in the electric double-layer at the metal-electrolyte interface, which has been proposed for thermally chargeable supercapacitors ${ }^{39}$. Aiming to understand the temperature-dependent potentials of the metals involved in our device, we measured changes in the open-circuit voltage for symmetric devices with two electrodes made of the same metals upon changes in temperature in the range from 22 to $38^{\circ} \mathrm{C}(\Delta T=$ $0-16 \mathrm{~K})$. The two identical electrodes were separated by a large distance (circa $5 \mathrm{~cm}$ ) and connected by an electrolyte bridge such that one electrode could be heated while the other was kept at room temperature. The results show that the surface potential of both $\mathrm{Au}$ and $\mathrm{Al}$ becomes more negative with increasing temperature (see Fig. 2a), which agrees with previous research ${ }^{40}$. In the studied temperature range $\left(22-38^{\circ} \mathrm{C}\right)$, the temperaturedependence of the surface potential is similar for the two metals, with values of $-3.4 \mathrm{mV} \mathrm{K}^{-1}$ for the Au electrode and $-4.7 \mathrm{mV} \mathrm{K}^{-1}$ 

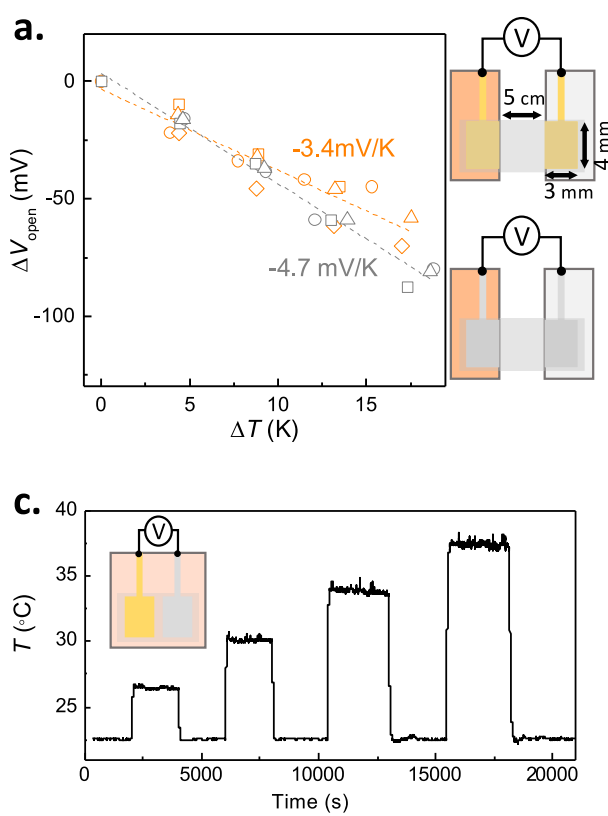

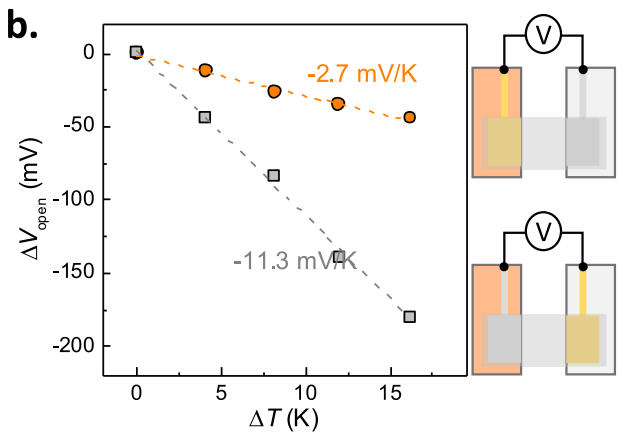

d.

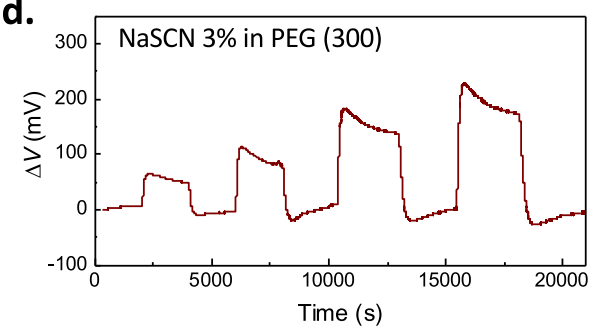

Fig. 2 Investigation of sensor mechanism by heating only one of the electrodes (heating is depicted by the orange shading). a Change in surface potential upon heating one of the electrodes for symmetric devices composed of two gold electrodes (yellow symbol shapes, results for four devices) or two aluminum electrodes (gray symbol shapes, results for three devices). b Same as in a but for an asymmetric device upon heating either the gold electrode (yellow) or the aluminum electrode (gray). c Controlled temperature changes applied to an EATS with a different electrolyte based on 3\% NaSCN in PEG (inset). d Open-circuit potential variations of the same device upon the heat cycles presented in c. All measurements were performed in a cleanroom at $\mathrm{RH}=30 \%$.

for the Al electrode. While previous research reported stronger temperature dependence for metals with larger work function (in aqueous solution), that was for a larger temperature range showing clear nonlinear behavior, and less variation between the different metals was reported in the lower temperature range similar to that investigated here ${ }^{40}$. We also note that there is no large temperature dependence of the capacitance of the $\mathrm{Au}$ or $\mathrm{Al}$ electrodes, with the Au having around twice capacitance/area (see Supplementary Fig. 4b). The fact that we do not see significant differences between the two metals in terms of change in potential with temperature indicates that alternative effects are responsible for the large signals of the asymmetric EATS.

In that respect, we note that the EATS resembles a galvanic cell $^{41}$, although without any redox couples added to the electrolyte. In order to investigate if the sensor signals of such non-ideal galvanic system depend on the electrolyte, we repeated the measurement using the same type of EATS device, but using a very different type of electrolyte (see the inset in Fig. 2c). This electrolyte was composed of sodium thiocyanate (NaSCN) in polyethylene glycol (PEG, $M_{\mathrm{w}}=300$ ), which is also stable during heating. Interestingly, we observed remarkably similar results when using the NaSCN:PEG electrolyte as when using the ionic liquid-based electrolyte, both in terms of sensitivity and time response (Fig. $2 \mathrm{~d}$ ). One thing that both electrolytes have in common is the presence of water and oxygen at small concentrations adsorbed from the atmosphere ${ }^{42,43}$, which could be related to the generated sensor signals. Regarding possible galvanic contribution to the signals, we note that EATS devices were stable for at least half a year (see Supplementary Figs. 5 and 11) and, hence, are not based on rapid consumption of the Al due to oxidation. Such long-term stability is advantageous for the concept to be used in practical applications.

The devices could also recover their open-circuit voltage after being electrically shorted for extended periods of time. In most galvanic cells, such action would quickly deplete the device and reduce the voltage to zero ${ }^{44}$. Another possibility is that the device functions as a hybrid supercapacitor ${ }^{45}$, with the Al electrode being most susceptible to electrochemical reactions while the Au acts more as a blocking electrode. Indeed, equivalent circuit analysis (see Supplementary Fig. 6) of impedance spectroscopy data for an EATS shows that the Al electrode provides a three orders of magnitude lower charge transfer resistance compared with the $\mathrm{Au}$ electrode in the studied temperature range (see Supplementary Table 1). Also, the Au electrode is almost purely capacitive while the Al electrode has a significant contribution from resistive processes (see details in Supplementary Note 2). To further elucidate this possible function of the EATS, we made asymmetric Au-Al devices (with similar electrode areas as for EATS) that were compatible with heating of only one of the electrodes at a time (see schematics in Fig. 2b). As shown in Fig. 2b, heating the Au electrode only resulted in modest changes in the open-circuit potential $\left(-2.7 \mathrm{mV} \mathrm{K}^{-1}\right)$. By contrast, heating the Al electrode induced large signals, with a sensitivity of $-11.3 \mathrm{mV} \mathrm{K}^{-1}$. This value is very similar to the response upon heating both electrodes of the full devices. Hence, these results confirm that the sensor signal is related to variation in temperature of the redox-active Al electrode in combination with being connected via the electrolyte to the more inert Au electrode.

Heat mapping by pixel arrays and plasmonic metasurfaces Motivated by good performance in terms of sensitivity, reproducibility, and stability, we investigate the EATS for heat mapping. Heat mapping could find use in many applications, including for e$\operatorname{skin}^{7,12,46}$, and we here demonstrate the concept for rectangular arrays with four sensor pixels.

We created a temperature gradient over the whole device by heating from one side while keeping the other side fixed at room temperature $\left(22^{\circ} \mathrm{C}\right.$, illustrated in Fig. 3a). The different pixels show different signal responses (Fig. $3 b$ ) and could accurately capture the respective local temperature variations as verified by commercial TCs close to (but not on) the pixels (Fig. 3c). We modified the device structure by replacing the flat metal film 
a.
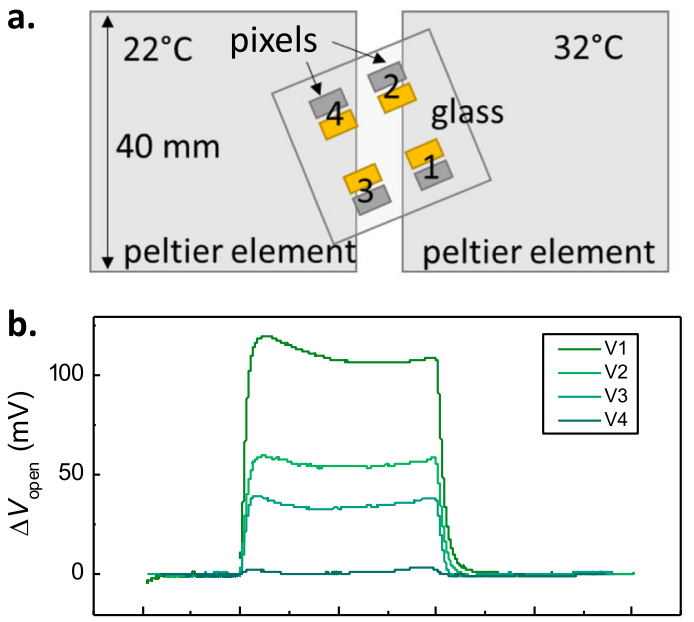

C.

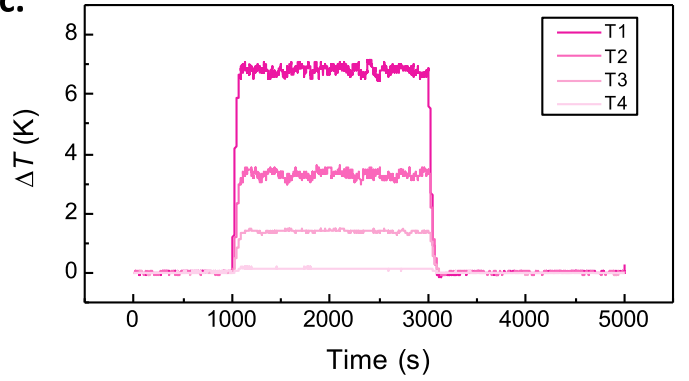

d.

e.
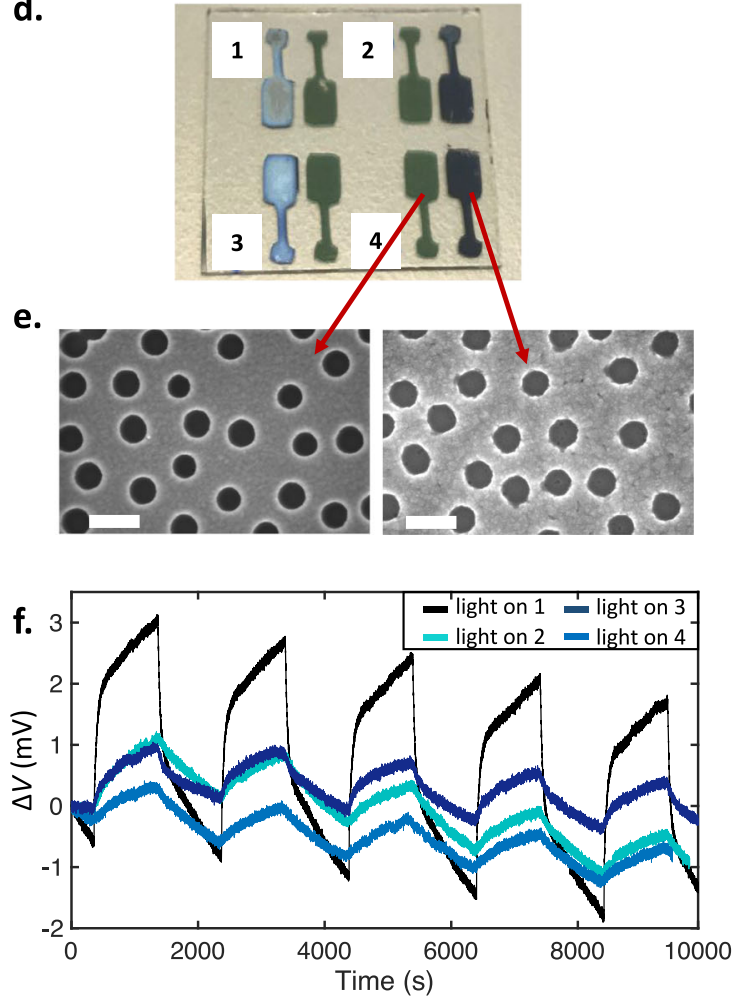

Fig. 3 Heat mapping using arrays of EATS and metasurface-based pixel arrays. a Schematic illustration of the sensor device and setup for non-uniform heating of the sample using two Peltier elements. The effective size of each Al/Au pixel is $24 \mathrm{~mm}^{2}$. b Voltage variations of the different pixels (V1-V4), at $\mathrm{RH}=30 \%$. c Temperature evolution of the pixels in $\mathbf{b}$ monitored by traditional TC placed close to the pixels (T1-T4). d Photograph of a 2 by 2 array of metasurface-modified pixels. e SEM images of a gold metasurface nanohole electrode (left) and an aluminum metasurface nanohole electrode (right). The scale bars represent $200 \mathrm{~nm}$. f Sensor response for pixel 1 upon illumination of the different plasmonic EATS by a solar simulator (at room humidity without control).

electrodes with the same type of electrodes but perforated with nanoholes. Using colloidal lithography ${ }^{47}$, we made sensor devices with $20 \mathrm{~nm}$ thick Al and Au nanohole electrodes, with $107 \mathrm{~nm}$ in diameter holes (see scanning electron microscopy (SEM) images in Fig. 3e). Such metal nanohole films strongly interact with and absorb light via excitation of plasmons (see absorption spectra in Supplementary Fig. 7) ${ }^{48}$, while their continuous metal structure still allows them to be used as electrodes ${ }^{33,49,50}$. The strong light absorption results in local heat generation, which makes the sensor pixels sensitive to light stimuli ${ }^{33,35,51}$. We used this lightsensitivity to individually stimulate different pixels by light, as an alternative methodology to heat mapping to evaluate the concept. We made 2 by 2 pixel arrays of plasmonic EATS (see Fig. 3d) and measured the response of one pixel (pixel 1) while varying which pixel that was illuminated (by a solar simulator, see Fig. 3f). As expected, the sensor signal was largest when illuminating the measured pixel (pixel 1) and decreased with distance between the measured and illuminated plasmonic pixels. This reflects the temperature distribution over the sample upon illumination at different positions. We also note that the sensor responded more rapidly for the illuminated pixel, which may be due to more rapid increase in temperature of the illuminated metasurface pixel compared to nearby areas.

Mitigating humidity effects and application as e-skin

For many applications, including e-skin, it is important to be able to decouple signals induced by different types of stimuli, such as changes in temperature and humidity. Measurements for heat cycles at different relative humidity (Fig. 4a) show that the sensitivity of the EATS remained high at both high $(\mathrm{RH}=30 \%$, green) and low ( $\mathrm{RH}=4 \%$, blue) relative humidity, with higher temperature response at low humidity. The sensor kinetics varied with humidity. At higher relative humidity, changes in temperature induced clear transient peaks (increase in $T$ ) and dips (decrease in 7 ) before saturation at somewhat lower signals. This behavior was less pronounced at low relative humidity. Furthermore, changes in relative humidity also caused significant changes in the open-circuit voltage when maintaining the device at a fixed temperature (dashed line in Fig. 4b). To further understand these effects, we performed impedance spectroscopy of an EATS at high and low relative humidities. Supplementary Fig. 8 presents the experimental results, which clearly show large variations in the electrical properties at different relative humidities. In particular, we found a large increase in the total resistance of the sensor when decreasing the relative humidity, indicating that an increase in the electrolyte bulk resistance and/or charge transfer resistance by lowering the relative humidity induces changes in the opencircuit potential of the sensor.

To decouple the signals induced by heat from those induced by relative humidity changes, we prepared a new type of encapsulated EATS. The metal electrodes were deposited on flexible polyethylene terephthalate (PET) foil, and after applying the electrolyte the whole device structure was encapsulated between two barrier films. As shown in Fig. 4b, the dependence of the open-circuit voltage to changes in relative humidity could be efficiently mitigated by encapsulation (solid line), showing no observable variations when alternating between relative humidity of $30 \%$ and $4 \%$.

Importantly, the encapsulated EATS devices provided high temperature response independent of relative humidity level, with sensitivity measured to $13.5 \mathrm{mVK}^{-1}$ at high relative humidity 

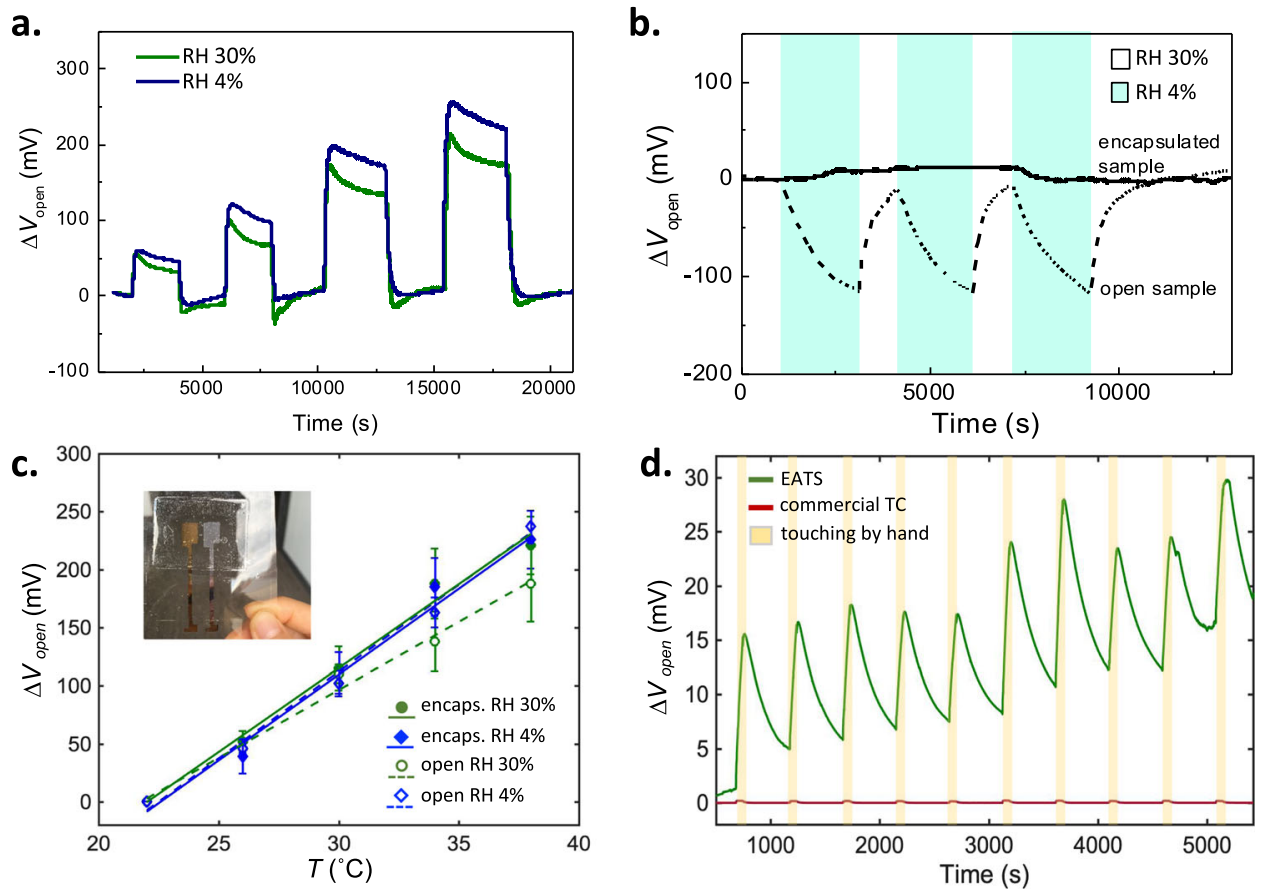

Fig. 4 Humidity response of EATS and mitigating the effect by encapsulation. a Temporal changes in open-circuit voltage of an EATS on a glass substrate during heat cycles (from 22 to $26^{\circ} \mathrm{C}, 30,34$, and $38^{\circ} \mathrm{C}$, respectively) at high humidity (green) and low humidity (blue). b Variation in open-circuit potential upon changes in humidity (alternating between $\mathrm{RH}=30 \%$ and $\mathrm{RH}=4 \%$ ) at $22^{\circ} \mathrm{C}$, for an open EATS (dashed line) and for an encapsulated EATS (solid line). c Changes in open-circuit voltage versus temperature at high (green) and low (blue) humidity for open (unfilled markers and dashed lines) and encapsulated EATS (filled markers and solid lines). Each data point (error bar) represents the average value (standard deviation) from three series of measurements. The inset shows a photograph of an encapsulated EATS device. $\mathbf{d}$ Change in open-circuit voltage generated by an encapsulated EATS (green) and a commercial TC (red) upon touch by a human hand (yellow shaded areas).

$(\mathrm{RH}=30 \%$, green filled markers in Fig. $4 \mathrm{c})$ and $13.4 \mathrm{mV} \mathrm{K}^{-1}$ at low humidity ( $\mathrm{RH}=4 \%$, blue filled markers). The open markers and dashed lines in Fig. 4c correspond to three measurements from two open EATS devices (without encapsulation). These open EATS devices exhibited different sensitivity at different humidity $\left(11.5 \mathrm{mV} \mathrm{K}^{-1}\right.$ at $\mathrm{RH}=30 \%$ and $13.8 \mathrm{mV} \mathrm{K}^{-1}$ at $\mathrm{RH}=4 \%$ ), while the encapsulated devices successfully eliminated the relative humidity dependence. In addition, the flexible encapsulated EATS also provided reproducible signals before and after repeated bending of the device (bent over a cylinder with diameter of $40 \mathrm{~mm}$, see details in Supplementary Note 7). Notably, some of these results were obtained 6 months after device preparation, demonstrating good long-term stability and mechanical properties of the encapsulated EATS devices.

Finally, we put our concept to practical test by evaluating its capability of detecting human touch, which forms one of the essential features when used in e-skin applications. Temperature changes from touch can be small, and we first investigated the response of EATS to small temperature changes and compared the results with those of a commercial TC. As shown in Supplementary Fig. 10, EATS could provide clear signals with high signal-to-noise ratios for small changes in temperature $\left(0.1^{\circ} \mathrm{C}\right)$, while the traditional TC was more limited and showed lower signal-to-noise ratios. Figure $4 \mathrm{~d}$ presents the variation in open-circuit voltage upon touch by a human hand (yellow shaded areas), both for an encapsulated EATS (green) and for a commercial TC (red). The commercial TC could only produce small signals on the order of $0.2 \mathrm{mV}$, while the EATS provided sensor signals of several millivolts, despite having more material to heat up. In terms of temperature range, the encapsulated EATS maintained its linear response also in a larger range from 14 to $55^{\circ} \mathrm{C}$ (Supplementary Fig. 11), making it suitable for many applications such as health-monitoring systems, and monitoring the temperature in packaging within this range.

In summary, we have reported a concept for ultrasensitive temperature sensing. The sensor signals are self-generated in analogy with traditional TCs, but with almost 300 times higher sensitivity. We show that the concept is applicable for heat mapping and that it can be made light-sensitive by modifying the sensor with light-absorbing plasmonic metasurfaces. While the sensor could also react to changes in humidity, we show that this feature can be eliminated by encapsulation when desired. The sensors were stable for at least several months and show good reproducibility between samples. Combined, the features and performance of the sensor make it suitable for use in applications such as e-skin, where the simplistic structure can be particularly useful for low-cost manufacturing at large scale.

\section{METHODS}

Fabrication of TC and (plasmonic/bendable) EATS

Glass wafers and $0.25 \mathrm{~mm}$-thick PET sheets were used as substrates for different devices. Glass wafers were rubbed in soap water and then ultrasonicated in soap water once and two times in deionized (DI) water for $5 \mathrm{~min}$ each. They were rinsed with acetone and isopropanol and dried with $\mathrm{N}_{2}$ stream. To remove organic contamination the glass substrates were surface-treated by UV-ozone for $15 \mathrm{~min}$. PET sheets were rinsed with DI water, acetone, and isopropanol and after drying with a $\mathrm{N}_{2}$ stream they were baked for $30 \mathrm{~min}$ at $130^{\circ} \mathrm{C}$ in an oven. Shadow mask evaporation was used to evaporate $20 \mathrm{~nm} \mathrm{Al}$ and $20 \mathrm{~nm}$ Au electrodes on the clean glass and PET substrates. For plasmonic EATS, we used colloidal lithography described elsewhere ${ }^{52}$ to fabricate $\mathrm{Al}$ and Au metasurface electrodes with thickness of $20 \mathrm{~nm}$ and nanohole dimeter of $107 \mathrm{~nm}$. To prepare the ionic liquid electrolyte for (plasmonic) EATS, 1-ethyl-3-methylimidazolium ethyl sulfate ([EMIM][ESO $\left.\left.{ }_{4}\right]\right)$ was mixed with HEC $(7.5 \mathrm{wt} \%)$ while heated to $100^{\circ} \mathrm{C}$ on a hotplate until forming a gel. Both chemicals were purchased 
from Sigma Aldrich. The electrolyte was then applied via a brush onto the electrodes, with thicknesses on the order of $300 \mu \mathrm{m}$ (measured for three samples by analyzing images taken using an optical contact angle and surface tension meter [KSV Instruments, CAM 200]).

NaSCN in PEG $\left(M_{\mathrm{w}}=300\right)$ was another electrolyte that we used to compare the function of the device with that of the $[E M I M]\left[E^{2} O_{4}\right]$ electrolyte. NaSCN was purchased from Sigma-Aldrich and mixed with PEG with $3 \mathrm{wt} \%$. It was applied on the devices in similar way as the[EMIM][ESO $\left.{ }_{4}\right]$ electrolyte.

Encapsulated devices were used to minimize the humidity dependence. The encapsulation was obtained by first attaching an adhesive barrier film to the back side of the device substrate. Al and Au electrodes were then deposited on PET substrate using shadow-mask thermal evaporation, with an electrode area of $5 \times 7 \mathrm{~mm}^{2}$. An adhesive plastic sheet was cut into the shape of a frame and attached on top of the device substrate, with the opening of the frame aligned to the Al and Au electrodes. The cavity inside the frame was filled with the ionic liquid electrolyte and the encapsulated device structure was completed by attaching another layer of the adhesive barrier film on top of the areas covered by electrodes and electrolyte on the front side of the device substrate. The adhesive polymer-based barrier film used for the encapsulation, HydroBlock AG2500, was purchased from Honeywell.

\section{Microstructure and optical characterization}

SEM (Zeiss, Sigma 500 Gemini) was used to study the distribution and quality of the Au and Al nanoholes. Absorption spectra of (plasmonic) EATS were evaluated using a custom-built setup consisting of a white light source ( $300 \mathrm{~W}$, Xe lamp, Newport), an integrating sphere (Labsphere), and an optical light guide connected to a spectrograph (Andor Shamrock 303i, Newton CCD detector).

\section{Impedance characterization}

The impedance measurements were carried out using an impedance spectrometer (Alpha high-resolution dielectric analyzer, Novocontrol Technologies $\mathrm{GmbH}$, Hundsangen, Germany). An AC voltage of $100 \mathrm{mV}$ was applied while sweeping the frequency from $1 \times 10^{6}$ to $1 \times 10^{-1} \mathrm{~Hz}$.

\section{Heat and radiation sensing evaluation}

Direct heat sensing measurements were performed by controlled heating of the samples from the bottom side via a Labview-controlled Peltier element $\left(22-40^{\circ} \mathrm{C}\right)$ and simultaneously monitoring the temperature on top of the devices using a TC. The open-circuit voltage generated by the devices was measured using a Keithley 2182A nanovoltmeter set to autorange. The Au electrodes were always connected to the positive measurement terminal of the nanovoltmeter if not stated otherwise. The temperature cycles were applied through a Labview program. Open-circuit voltage measurements throughout this work were performed in a cleanroom with relative humidity of $30 \%$ unless otherwise stated. To study the effect of humidity on the response of the devices, the humidity of the measurement chamber was reduced to $\sim 4 \%$ by constant purging of $\mathrm{N}_{2}$. Monitoring of the radiation-induced heating was carried out using a solar simulator (LCS-100, Oriel Instruments, $300 \mathrm{~W}$ Xe lamp, AM1.5Gfilter) calibrated to an irradiance of 1 Sun $\left(1000 \mathrm{~W} \mathrm{~m}^{-2}\right)$ and a custom-designed aperture to only illuminate only one pixel at time. Using an optical beam shutter (SH, Thorlabs), light on and off states were controlled. Via a Labview-controlled Keithley 2182A nanovoltmeter, the open-circuit voltage generated by the devices was measured.

\section{Evaluating effects on bending}

The sensor response of an encapsulated EATS was acquired before and after repeated bending of the device around a rod with diameter of $40 \mathrm{~mm}$. For each bending cycle, the device was bent and unbent 10 times before being placed on the Peltier element to measure the open-circuit potential upon temperature cycles. This whole bending and measurement procedure was repeated on three consecutive days.

\section{DATA AVAILABILITY}

The data that support the findings of this study are available from the corresponding authors upon reasonable request.
Received: 10 March 2020; Accepted: 19 August 2020; Published online: 15 September 2020

\section{REFERENCES}

1. Verhage, L., Angenent, G. C. \& Immink, R. G. H. Research on floral timing by ambient temperature comes into blossom. Trends Plant Sci. 19, 583-591 (2014).

2. Poff, K. L. Temperature Sensing in Microorganisms. In Sensory Perception and Transduction in Aneural Organisms (eds Colombetti, G., Lenci, F. \& Song, P.-S.). 299-307 (Springer US, 1985).

3. Lister, T., Wright, P. A. \& Chappell, P. H. Optical properties of human skin. J. Biomed. Opt. 17, 1-15 (2012).

4. Pogorzala, L. A., Mishra, S. K. \& Hoon, M. A. The cellular code for mammalian thermosensation. J. Neurosci. 33, 5533-5541 (2013).

5. Lee, W. W. et al. A neuro-inspired artificial peripheral nervous system for scalable electronic skins. Sci. Robot. 4, eaax2198-eaax2213 (2019).

6. Byun, J. et al. Electronic skins for soft, compact, reversible assembly of wirelessly activated fully soft robots. Sci. Robot. 3, eaas9020-eaas9020 (2018).

7. Hua, Q. et al. Skin-inspired highly stretchable and conformable matrix networks for multifunctional sensing. Nat. Commun. 9, 244-255 (2018).

8. Chou, H. H. et al. A chameleon-inspired stretchable electronic skin with interactive colour changing controlled by tactile sensing. Nat. Commun. 6, 1-10 (2015).

9. Wang, S., Oh, J. Y., Xu, J., Tran, H. \& Bao, Z. Skin-inspired electronics: an emerging paradigm. Acc. Chem. Res. 51, 1033-1045 (2018).

10. $\mathrm{Gu}, \mathrm{Y}$. et al. Mini review on flexible and wearable electronics for monitoring human health information. Nanoscale Res. Lett. 14, 263-263 (2019).

11. Zhang, Y. et al. Dual-mode electronic skin with integrated tactile sensing and visualized injury warning. ACS Appl. Mater. 9, 37493-37500 (2017).

12. Zhang, $C$. et al. A stretchable dual-mode sensor array for multifunctional robotic electronic skin. Nano Energy 62, 164-170 (2019).

13. Zhao, S. \& Zhu, R. Electronic skin with multifunction sensors based on thermosensation. Adv. Mater. 29, 1606151-1606151 (2017).

14. Ma, Z. et al. Advanced electronic skin devices for healthcare applications. J. Mater. Chem. B 7, 173-197 (2019).

15. Someya, T. et al. Conformable, flexible, large-area networks of pressure and thermal sensors with organic transistor active matrixes. Proc. Natl Acad. Sci. USA 102, 12321-12325 (2005).

16. Hasan, S. A. et al. ZnO thin film based flexible temperature sensor. In 2017 IEEE Sensors, Glasgow. 1-3. https://doi.org/10.1109/ICSENS.2017.8234387 (IEEE, 2017).

17. García Núñez, C., Manjakkal, L. \& Dahiya, R. Energy autonomous electronic skin. npj Flex. Electron. 3, 1-24 (2019).

18. Chortos, A. \& Bao, Z. Skin-inspired electronic devices. Mater. Today 17, 321-331 (2014).

19. Lee, M. H., Guo, R. \& Bhalla, A. S. Pyroelectric sensors. J. Electroceram. 2, 229-242 (1998).

20. Whatmore, R. W. \& Watton, R. In Infrared Detectors and Emitters: Materials and Devices (eds Capper, P. \& Elliott, C. T.) 99-147 (Springer US, 2001).

21. Shiran Chaharsoughi, M. et al. Hybrid plasmonic and pyroelectric harvesting of light fluctuations. Adv. Opt. Mater. 6, 1701051-1701051 (2018).

22. Gold, H. et al. Flexible single-substrate integrated active-matrix pyroelectric sensor. Phys. Status Solidi (RRL)—Rapid Res. Lett. https://doi.org/10.1002/pssr.201900277 (2019).

23. Sirusi, A. A. \& Ross, J. H. In Annual Reports on NMR Spectroscopy Webb, Vol. 92 (ed Graham, A. B. T.) 137-198 (Academic Press, 2017).

24. Han, N. \& Ho, J. C. In Nanocrystalline Materials (ed Tjong, S.-C.) 75-120, https://www. sciencedirect.com/science/article/pii/B9780124077966000038 (Elsevier, 2014).

25. Rao, J. et al. Recent progress in self-powered skin sensors. Sensors 19, 2763-2782 (2019).

26. Zhao, D. et al. Polymer gels with tunable ionic Seebeck coefficient for ultrasensitive printed thermopiles. Nat. Commun. 10, 1093-1101 (2019).

27. Costesèque, P., Mojtabi, A. \& Platten, J. K. Thermodiffusion phenomena. CR Méc. 339, 275-279 (2011).

28. Rahman, M. T. et al. High performance flexible temperature sensors via nanoparticle printing. ACS Appl. Nano Mater. 2, 3280-3291 (2019).

29. Bajpai, P. In Biermann's Handbook of Pulp and Paper (ed. Bajpai, P.) 483-492 (Elsevier, 2018).

30. Zhao, D., Fabiano, S., Berggren, M. \& Crispin, X. lonic thermoelectric gating organic transistors. Nat. Commun. 8, 14214-14214 (2017).

31. Baffou, G. \& Quidant, R. Thermo-plasmonics: using metallic nanostructures as nano-sources of heat. Laser Photon. Rev. 7, 171-187 (2013).

32. Baffou, G., Quidant, R. \& Girard, C. Heat generation in plasmonic nanostructures: influence of morphology. Appl. Phys. Lett. 94, 1-3 (2009).

33. Tordera, D., Zhao, D., Volkov, A. V., Crispin, X. \& Jonsson, M. P. Thermoplasmonic semitransparent nanohole electrodes. Nano Lett. 17, 3145-3151 (2017).

34. Shiran Chaharsoughi, M. et al. Hybrid plasmonic and pyroelectric harvesting of light fluctuations. Adv. Opt. Mater. 6, 1701051-1701058 (2018). 
35. Shiran Chaharsoughi, M., Zhao, D., Crispin, X., Fabiano, S. \& Jonsson, M. P. Thermodiffusion-assisted pyroelectrics-enabling rapid and stable heat and radiation sensing. Adv. Funct. Mater. https://doi.org/10.1002/adfm.201900572 (2019).

36. Brooke, R. et al. Supercapacitors on demand: all-printed energy storage devices with adaptable design. Flex. Print. Electron. 4, 15006-15006 (2019).

37. Salam, B., Shan, X. C., Zhanhong, C. \& Lok, B. K. Multilayer Roll-to-Roll ScreenPrinting for Printed Electronics Applications. In 2018 IEEE 20th Electronics Packaging Technology Conference (EPTC). 359-362. https://doi.org/10.1109/ EPTC.2018.8654393 (Singapore, Singapore, 2018).

38. Carlos Montalvo, K. D., Tommy Höglund, Svensson, Magnus, Maialen \& Oribe, R. B. A. M. B. In Policy Assessment Demonstrator ACREO Printed Electronics Arena, 84 (Multi Key Enabling Technologies Pilot Lines, 2014).

39. Lim, H., Lu, W., Chen, X. \& Qiao, Y. Effects of ion concentration on thermally chargeable double-layer supercapacitors. Nanotechnology 24, 3-8 (2013).

40. Lim, H. et al. Effects of work function on thermal sensitivity of electrode potential. Appl. Phys. Lett. 106, 223901-223906 (2015).

41. Vincent, C. A. In Modern Batteries (eds Vincent, C. A. \& Scrosati, B. B. T.) 18-64 (Butterworth-Heinemann, 1997).

42. Bartkowiakb, G. \& Frydrych, I. In Handbook of Medical Textiles (ed Bartels, V. T.) 505-546 (Woodhead Publishing, 2011).

43. Baird, J. A., Olayo-Valles, R., Rinaldi, C. \& Taylor, L. S. Effect of molecular weight, temperature, and additives on the moisture sorption properties of polyethylene glycol. J. Pharm. Sci. 99, 154-168 (2010).

44. Baboian, R. In ASM Handbook, Volume 13A: Corrosion: Fundamentals, Testing, and Protection (ASM International, 2003).

45. Muzaffar, A., Ahamed, M. B., Deshmukh, K. \& Thirumalai, J. A review on recent advances in hybrid supercapacitors: design, fabrication and applications. Renew. Sust. Energy Rev. 101, 123-145 (2019).

46. Webb, R. C. et al. Ultrathin conformal devices for precise and continuous thermal characterization of human skin. Nat. Mater. 12, 1078-1078 (2013).

47. Hanarp, P., Sutherland, D., Gold, J. \& Kasemo, B. Nanostructured model biomaterial surfaces prepared by colloidal lithography. Nanostruct. Mater. 12, 429-432 (1999).

48. Kang, E. S. H., Ekinge, H. \& Jonsson, M. P. Plasmonic fanoholes: on the gradual transition from suppressed to enhanced optical transmission through nanohole arrays in metal films of increasing film thickness. Opt. Mater. Express 9, 1404-1404 (2019).

49. Jonsson, M. P., Jönsson, P. \& Höök, F. Simultaneous nanoplasmonic and quartz crystal microbalance sensing: analysis of biomolecular conformational changes and quantification of the bound molecular mass. Anal. Chem. 80, 7988-7995 (2008).

50. Shiran Chaharsoughi, M., Zhao, D., Crispin, X., Fabiano, S. \& Jonsson, M. P. Thermodiffusion-assisted pyroelectrics-enabling rapid and stable heat and radiation sensing. Adv. Funct. Mater. 29, 1900572-1900581 (2019).

51. Pan, Y. et al. A rapid response thin-film plasmonic-thermoelectric light detector. Sci. Rep. 6, 37564 (2016).

52. Fredriksson, H. et al. Hole-mask colloidal lithography. Adv. Mater. 19, 4297-4302 (2007).

\section{ACKNOWLEDGEMENTS}

The authors gratefully acknowledge financial support from the Swedish Foundation for Strategic Research, the Swedish Research Council, the Wenner-Gren Foundations,
Essity, the Swedish Government Strategic Research Area in Materials Science on Functional Materials at Linköping University (Faculty Grant SFO-Mat-LiU No. 2009 00971) and the Vinnova Digital Cellulose Competence Center (DCC), Diary number 2016-05193. The work was also supported by Treesearch.se. Open access funding provided by Linköping University.

\section{AUTHOR CONTRIBUTIONS}

M.P.J. and D.Z. conceived the project and supervised the research. M.S.C. performed the device fabrication and took part in measurements and data analysis. P.A.E. and M. S.C. performed sample preparations on flexible substrates and encapsulation of the devices. D.Z. and M.S.C. performed experiments on the humidity and temperature sensing properties of the devices. J.E. conducted the impedance spectroscopy measurements and corresponding data analysis. M.S.C. wrote the paper with contributions from all authors.

\section{COMPETING INTERESTS}

The authors declare no competing interests.

\section{ADDITIONAL INFORMATION}

Supplementary information is available for this paper at https://doi.org/10.1038/ s41528-020-00086-5.

Correspondence and requests for materials should be addressed to D.Z. or M.P.J.

Reprints and permission information is available at http://www.nature.com/ reprints

Publisher's note Springer Nature remains neutral with regard to jurisdictional claims in published maps and institutional affiliations.

\begin{abstract}
(c) (i)
Open Access This article is licensed under a Creative Commons Attribution 4.0 International License, which permits use, sharing, adaptation, distribution and reproduction in any medium or format, as long as you give appropriate credit to the original author(s) and the source, provide a link to the Creative Commons license, and indicate if changes were made. The images or other third party material in this article are included in the article's Creative Commons license, unless indicated otherwise in a credit line to the material. If material is not included in the article's Creative Commons license and your intended use is not permitted by statutory regulation or exceeds the permitted use, you will need to obtain permission directly from the copyright holder. To view a copy of this license, visit http://creativecommons. org/licenses/by/4.0/.
\end{abstract}

(c) The Author(s) 2020 